\title{
Folgen einer Partynacht
}

Anne Frederike Wunder, Michael Oelckers, Guntram Lock

Verätzungen des Ösophagus entstehen üblicherweise akzidentell oder in suizidaler Absicht.

Sie können zu schweren akuten und chronischen Krankheitsbildern führen: Neben lebenslangen

Dysphagiebeschwerden durch rezidivierende Strikturen besteht mittel- und langfristig auch ein erhöhtes Risiko für eine Karzinomentwicklung. Wir berichten hier über den ungewöhnlichen Fall einer ausgeprägten Ösophagusverätzung nach irrtümlichem Trinken einer „Schnüffel“-Partydroge.

Anamnese | Eine 18-jährige Patientin wurde nach einer ambulanten Ösophagoskopie unter der Diagnose einer schweren und nicht passierbaren hämorrhagischen Ösophagitis als Notfall eingewiesen. In den vorangegangenen 3 Wochen war für die Patientin die Aufnahme fester Nahrung immer mühsamer geworden und in den letzten Tagen gar nicht mehr möglich gewesen. Zuletzt konnte sie auch flüssige Speisen und selbst Speichel nur noch mit größerer Anstrengung und unter Schmerzen schlucken. Innerhalb von 3 Wochen hatte sie $8 \mathrm{~kg}$ Körpergewicht verloren.

Ursache der Beschwerden | Auf genaueres Nachfragen gab die Patientin an, dass sie vor Beginn der Symptomatik vom Freund eines Freundes auf einer Party ein Fläschchen mit einem ihr unbekannten Inhalt erhalten habe und zum Probieren aufgefordert worden sei. Statt die Dämpfe des Inhalts zu inhalieren, habe sie aus Unkenntnis bezüglich der Anwendung einen Schluck aus dem Fläschchen getrunken. Nach der Ingestion wäre sie für einen kurzen Moment bewusstlos geworden. Aufgrund der Synkope sei sie in die Notaufnahme einer nahegelegenen Klinik eingeliefert worden. In der Notaufnahme seien zunächst abdominelle Krämpfe aufgetreten. Nach Abklingen der Symptome sei sie ohne weitere spezifische Diagnostik aus der dortigen Notaufnahme entlassen worden.

Körperliche Untersuchung I Zum Zeitpunkt der Aufnahme befand sich die sehr schlanke Patientin (BMI $16,6 \mathrm{~kg} / \mathrm{m}^{2}$ ) in einem schmerzbedingt leicht reduzierten Allgemeinzustand.

- Die Herzfrequenz war mit 102 Schlägen / Minute erhöht.

- Der Blutdruck lag bei 119/67 mmHg.

- Der internistische Untersuchungsbefund war ansonsten, bis auf einen leichten epigastrischen Druckschmerz und spärliche Darmgeräuschen, nicht wegweisend.

Laborbefunde I Zum Zeitpunkt der Aufnahme waren die Laborwerte - bis auf ein geringgradig erhöhtes CRP (29,75 mg/l) - unauffällig.
Endoskopie I Ösophagoskopisch wurde eine massive Ösophagusverätzung festgestellt, die knapp unterhalb des oberen Ösophagussphinkters (ca. $20 \mathrm{~cm}$ ab Zahnreihe) begann. Bei ca. $40 \mathrm{~cm}$ fand sich eine höchstgradige Stenose: Selbst mit einem ultradünnen flexiblen Gastroskop (4,9 mm Durchmesser) konnte diese nicht passiert werden ( Abb. 1). Der histologische Befund einer Biopsie aus dem mittleren Drittel des Ösophagus passte zu einer chemisch-induzierten Schleimhautschädigung.

Initiale Therapie | Während der initialen Endoskopie wurde unter Durchleuchtung eine Drahtpassage durchgeführt. Der Patientin wurde anschließend eine 12-French-Ernährungssonde in Seldinger-Technik eingelegt. Über diese wurde sie zunächst enteral ernährt. 3 Tage später wurde die

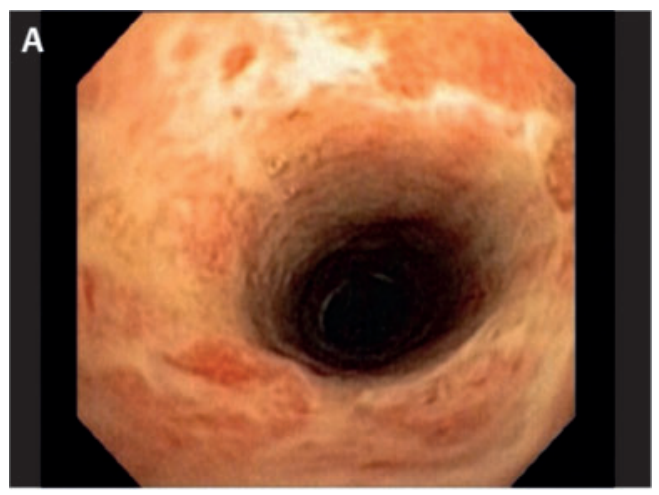

Abb. 1 (A) und (B) Ösophagoskopie 3 Wochen nach Ingestion: Schwere chemisch induzierte hämorrhagische Ösophagitis.

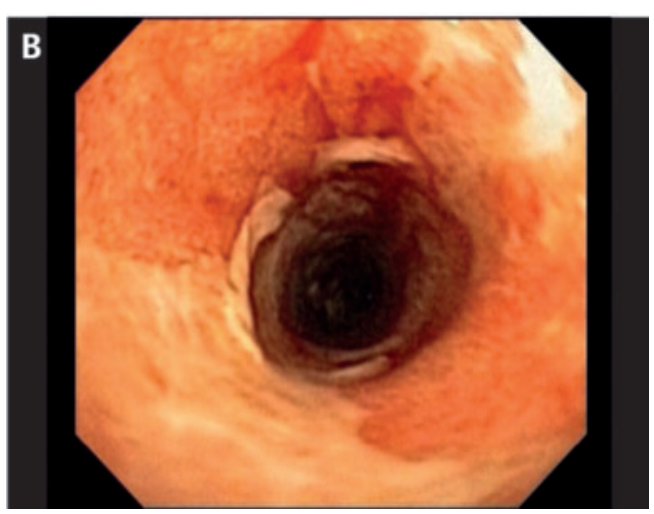


Abb. 2 (A) und (B) Ösophagoskopie ca. ein halbes Jahr später: Restenosierung durch Narbenbildung.
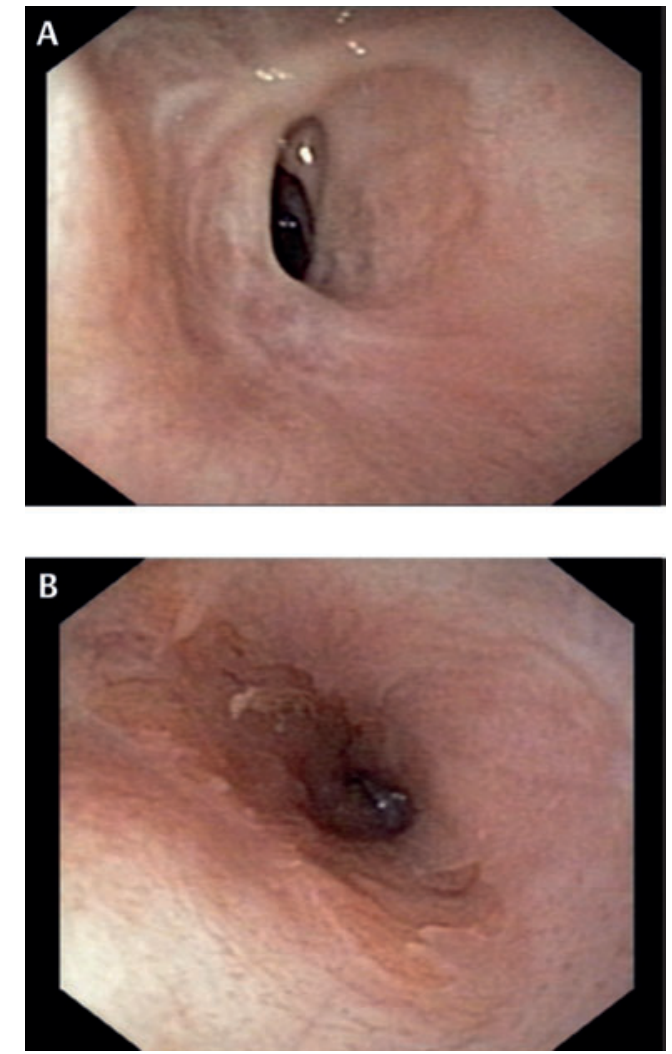

Abb. 3 Ösophagoskopie nach 3 Jahren: Langstreckig vernarbter Ösophagus.

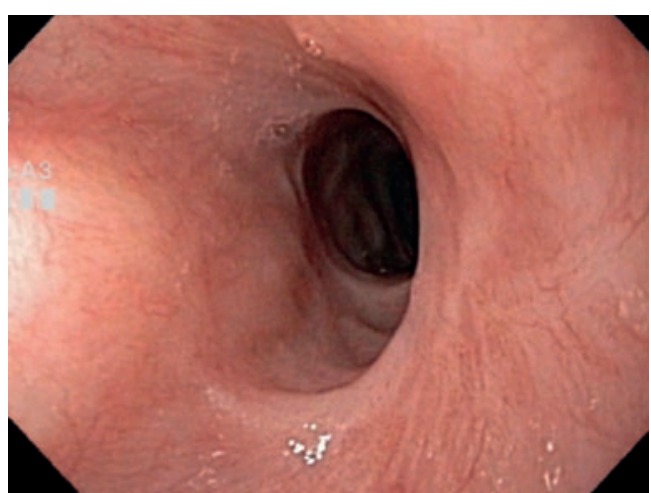

Sonde entfernt. Daraufhin konnte die distale Ösophagusstenose mit dem 4,9 mm Gastroskop passiert werden. Der Magen war endoskopisch-makroskopisch unauffällig. Mit Savary-Bougies gelang es, die Speiseröhre der Patientin zu dilatieren beginnend mit einem Savary-Bougie von $5 \mathrm{~mm}$ bis hin zu einem Durchmesser von $9 \mathrm{~mm}$. Anfänglich war das Behandlungsergebnis gut: Der Ösophagus weitete sich durch Bougieren schrittweise auf $11 \mathrm{~mm}$.

Therapie im Verlauf | 5,5 Monate später diagnostizierten wir eine Restenosierungstendenz mit z.T. ausgeprägten Narbenbildungen ( $\triangleright$ Abb. 2 ). Durch Nadelmesser-Inzisionen in der Longitudinalachse des Ösophagus und Steroidinjektionen mit Triamcinolon ließen sich die Narben schließlich mit gutem Ergebnis behandeln (Übersicht zu entsprechenden Techniken unter [1]). Nach zunächst über 2-jähriger Beschwerdefreiheit stellte sich die Patientin mit wiederaufgetretener Dys- phagie beim Schlucken von festen Speisen aufgrund einer Ösophagusenge vor. Ihre Speiseröhre musste erneut stufenweise von $11,8 \mathrm{~mm}$ auf $15 \mathrm{~mm}$ bougiert werden. Auch 3 Jahre nach der ersten Bougierung war der Ösophagus noch langstreckig mäßig stenosiert und vernarbt $(\triangleright$ Abb. 3 ). Seit 2 Jahren, insgesamt 5 Jahre nach der ersten Behandlung, ist die Patientin nun anhaltend beschwerdefrei.

\section{Diskussion}

Beliebte Partydroge I Wie sich nach Recherchen im Umfeld der Patientin herausstellte, handelte es sich bei dem konsumierten Inhalt des Fläschchens um die seit den 1970er Jahren beliebte Party- und Schnüffeldroge Poppers. Der Name Poppers leitet sich von dem Geräusch ab, das beim Öffnen der Glasfläschchen entsteht (to pop - knallen), in denen die Substanzen ehemals erhältlich waren. Auch die Schraubverschlüsse der heutigen bunten Plastikfläschchen „ploppen“ aufgrund des höheren Drucks in der Flasche beim Öffnen hörbar [2]. In der Szene sind Poppers auch unter den Namen „flüssiges Gold“, „Rave“, „Snappers“ oder „Rush“ bekannt.

Konsum ist legal | Obwohl sie als Schnüffeldroge verwendet werden, unterliegen Poppers nicht dem Betäubungs-, sondern aufgrund der ursprünglichen Herkunft des Stoffes dem Arzneimittelgesetz. Der Besitz und Konsum ist in Deutschland dadurch legal. Da der Handel jedoch gegen das Arzneimittelgesetz verstößt, werden Poppers unter Angaben falscher Verwendungszwecke, etwa als „Reinigungsmittel“, „Video-Tonkopfreiniger“, „Zimmerduft“ oder „Lederputzmittel“ bereits für 5-10€ pro Flasche verkauft.

Nach einer aktuellen französischen Arbeit ist der Konsum von Poppers zwischen den Jahren 2000 und 2010 erheblich angestiegen.

Laut dieser Arbeit haben über 5\% der 18-64-Jährigen zu irgendeinem Zeitpunkt ihres Lebens diese Droge konsumiert. Damit sei sie nach Cannabis die am zweithäufigsten verwendete Droge [3].

Ursprünglich angewandt bei Angina pectoris | Die bei Raumtemperatur gelbe, flüssige, leicht flüchtige Schnüffeldroge hat einen fruchtigen und süßen Geruch.

Der Wirkstoff ist meist ein organisches Nitrit, wie Amylnitrit, Butylnitrit, Isoamylnitrit und Isopropylnitrit, oder ein entsprechendes Gemisch dieser Nitrite.

Organische Nitrite wie Amylnitrit wirken als Stickstoff-Donatoren. Sie wurden Jahrzehnte zur 
Behandlung der Angina pectoris verwendet, jedoch aufgrund ihrer kurzen Wirkdauer (wenige Minuten) später durch andere Medikamente ersetzt [2].

Kurzer Rausch mit starken Nebenwirkungen | Das Schnüffeln von Poppers führt zu einer kurzen relaxierenden Wirkung an der gatten Muskulatur und zu einer Vasodilatation. Es resultiert ein etwa 5 bis 10-minütiger Rauschzustand von Zeitlosigkeit, einhergehend mit

- Hitzegefühl,

- Muskelentspannung,

- vermindertem Schmerzempfinden,

- sexueller Luststeigerung und

- Hemmungslosigkeit.

Die Dauer des Rausches ist abhängig von der inhalierten Menge [4]. Die Party- und Sexdroge Poppers ist insbesondere in homosexuellen Kreisen beliebt, da sie aufgrund der muskelrelaxierenden Wirkung auf den Schließmuskel den Analverkehr erleichtert [5].

Cave Nebenwirkungen von Poppers:

- Tachykardie

- Hypotension

- Schwindel

- Hitzewallungen

- Nausea/Erbrechen

- Nitritinduzierte Cephalgien

- Methämoglobinämie [6, 7] und hämolytische Anämie [8, 9, 10]

Bei wiederholten Anwendungen:

- Dermatitis [11]

- Makulopathie [12]

- Visusverlust [13]

Schwere Verätzungen möglich | Im Internet wird immer wieder vor dem Kontakt von Poppers mit Schleimhäuten gewarnt. Schwere Verätzungen durch Inhalation oder gar Ingestion von Poppers lassen sich auf die Herstellung mit salpetriger Säure zurückführen [14, 15].

Zunehmender Konsum - häufigere Unfälle? I Ein ähnlicher Fall wie der von uns berichtete ist in der medizinischen Fachliteratur nicht zu finden (Pubmed recherche mit [„,alkyl nitrite“ OR „amyl nitrite“ OR „poppers“] AND [,esophagus“ OR „stricture“]). Lediglich in der Laienpresse ist (neben der Beschreibung unseres Falles) ein tödlich endender Fall zu finden: Eine 21-jährige Frau hatte in einer Diskothek ebenfalls aus Unwissenheit Poppers getrunken, statt die Dämpfe zu inhalieren. Sie starb wenige Tage danach im Krankenhaus [16]. Aufgrund des offenbar erheblich zunehmenden Gebrauchs dieser Droge dürfte auch die Wahrscheinlichkeit versehentlicher oraler Ingestionen mit den hier beschriebenen fatalen Konsequenzen ansteigen.

\section{Konsequenz für Klink und Praxis}

- Bei Ingestion von flüssigen Partydrogen unbekannten Inhalts ist daran zu denken, dass es sich um die versehentliche Ingestion eines „Schnüffelstoffes“ gehandelt haben könnte.

- Initiale Symptome sind hierbei oft unspezifisch. Eine ausführliche Anamnese, - ggf. Fremdanamnese - ist daher unbedingt notwendig.

- Eine versehentliche orale Ingestion von Poppers ist ein akuter Notfall und kann zu schweren, akut lebensbedrohlichen oder mit schweren Spätfolgen assoziierten Ösophagusverätzungen führen.

\section{Literatur}

1 van Boeckel PG, Siersema PD. Refractory esophageal strictures: what to do when dilation fails. Curr Treat Options Gastroenterol 2015; 13: 47-58

2 DocCheck Flexikon. Poppers. http://flexikon. doccheck.com/de/Poppers (letzter Zugriff 03.09.2015)

3 Beck F, Guignard R, Richard JB. Poppers at top. Med Sci 2014; 30: 916-921

4 News.de. Zur Extase geschnüffelt (30.11.2011). http://www.news.de/reisen-undleben/855244035/ zur-ekstase-geschnueffelt/1/ (letzter Zugriff 03.09.2015)

5 Romanelli F, Smith KM, Thornton AC et al. Poppers: epidemiology and clinical management of inhaled nitrite abuse. Pharmacotherapy 2004; 24: 69-78

6 Forsyth R], Moulden A. Methaemoglobinaemia after ingestion of amyl nitrite. Arch Dis Child 1991; 66: 152

7 Edwards RJ, Ujma J. Extreme methaemoglobinaemia secondary to recreational use of amyl nitrite. J Accid Emerg Med 1995; 12: 138-142

8 Graves TD, Mitchell S. Acute haemolytic anaemia after inhalation of amyl nitrite. J R Soc Med 2003; 96: 594-595

9 Shortt J, Polizzotto MN, Opat SS et al. Oxidative haemolysis due to ,poppers'. Br 」 Haematol 2008; 142: 328

10 Neuberger A, Fishman S, Golik A. Hemolytic anemia in a G6PD-deficient man after inhalation of amyl nitrite („poppers“). Isr Med Assoc ] 2002; 4: 1085-1086

11 Fisher AA, Brancaccio RR, Jelinek JE. Facial dermatitis in men due to inhalation of butyl nitrite. Cutis 1981; 27: 146, 152-153

12 Gruener AM, Jeffries MA, El Housseini Z et al. Poppers maculopathy. Lancet 2014; 384: 1606

13 Independent Scientific Committee on Drugs. Alkyl Nitrites /,Poppers'. http://www.drugscience.org. uk/drugs-info/alkyl-nitrites-poppers/ (letzter Zugriff 03.09.2015)

14 http://www.chemie.de/lexikon/Amylnitrit.html (letzter Zugriff 03.09.2015)

15 The University of Kansas Hospital. Poison Facts: High Chemicals: Nitric Acid. http://www.kumed. com/ /media/Imported/kumed/documents/ kdhenitricacid.ashx (letzter Zugriff 03.09.2015)

16 Favez J, Marty S. Poppers geschluckt statt inhaliert (18.01.2012). http://www.20min.ch/news/kreuz und_quer / story / Poppers-geschluckt-statt-gesnifft-24279453 (letzter Zugriff 03.09.2015)

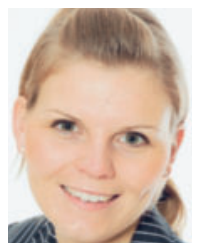

Anne Frederike Wunder ist Assistenzärztin der Klinik für Innere Medizin mit Schwerpunkt Gastroenterologie/ Hepatologie, Albertinen-Krankenhaus Hamburg

Anne.wunder@albertinen.de

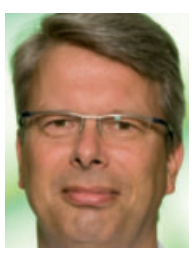

Michael Oelckers ist Oberarzt der Klinik für Innere Medizin mit Schwerpunkt Gastroenterologie/Hepatologie, Albertinen-Krankenhaus Hamburg

michael.oelckers@ albertinen.de

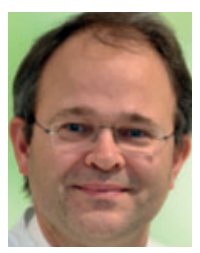

Prof. Dr. Guntram Lock ist Chefarzt der Klinik für Innere Medizin mit Schwerpunkt Gastroenterologie/Hepatologie Albertinen-Krankenhaus Hamburg guntram.lock@albertinen.de

Interessenkonflikt

Die Autoren geben an, dass kein Interessenkonflikt besteht.

DOI 10.1055/s-0041-106022

Dtsch Med Wochenschr 2015; 140: 1611-1613 (c) Georg Thieme Verlag KG . Stuttgart · New York . ISSN 0012-0472 